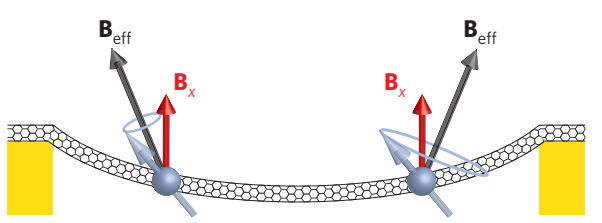

Figure 2 | The effective magnetic field $\mathbf{B}_{\text {eff }}$ in a bent nanotube is the sum of the external field $\mathbf{B}_{x}$ and a field produced by the spin-orbit interaction $\mathbf{B}_{\text {so }}$ (ref. 4). While $\mathbf{B}_{x}$ is constant along the tube, $\mathbf{B}_{\text {so }}$ points along the local (axial) direction of the nanotube and thus varies along the tube. If an electron is moved back and forth along the tube using an alternating electric field, then the electron spin (blue arrow) is exposed to an alternating magnetic field $\mathbf{B}_{\text {eff }}(t)=\mathbf{B}_{x}+\mathbf{B}_{\mathrm{so}}(t)$, where $t$ denotes time, which rotates the spin (electric dipole resonance).

which two electrons with the same spin (spin triplet) occupy the same dot, that is, if they have different valley indices. In a GaAs quantum dot, alternative states also exist, but usually only at a much higher energy. A way around the problem is to consider a combined valley-spin blockade, rather than a pure spin one ${ }^{5}$. In a nanotube, the two valleys and spin orientations lead to four states. Owing to the spin-orbit interaction, these states are split into two pairs (Fig. 1c). Previous experiments ${ }^{6,7}$ have observed distinct signatures in the electric current for which the valley-spin blockade is responsible, but the small energy gaps in nanotube quantum dots provide easy access to additional states at relatively low energy, thus making the observation of spin blockade rather difficult. To render the valley-spin blockade more robust, Pei et al. tune their semiconducting nanotube double dot into an ambipolar regime, where the states in the right dot are just below the bandgap. This means that there are no other available states spoiling the blockade within a large energy range.

The researchers were then able to induce spin rotations through the socalled electric-dipole spin resonance. They exploited the fact that the spin-orbit interaction leads to an effective magnetic field along the axial direction of the tube ${ }^{8}$ and its direction changes when an electron moves along a bent tube (Fig. 2). If an alternating electric field is used to shake the electron back and forth along the tube, an effective alternating magnetic field is produced, which then rotates the spin ${ }^{9}$. One reason why this works so well is that the spin-orbit interaction in the nanotubes studied by Pei and colleagues ${ }^{4}$ is stronger than in tubes investigated earlier, for reasons that at this stage seem unclear.
The applied oscillatory electric field in fact couples to both the spin and the valley degrees of freedom in a bent tube ${ }^{9}$, whereas in disordered straight tubes, pure valley rotations are possible $\mathrm{e}^{10}$. It would be interesting if pure spin rotations could be achieved as well, and if spin and valley operations could be distinguished using the valley-spin blockade. After all, the valley index is a two-valued degree of freedom, and one may wonder if it is any good as a bit, or even a qubit. Finally, valley degeneracy occurs in other materials with low nuclear-spin content, such as silicon and germanium, which likewise are potential host materials for spin qubits.

Guido Burkard is at the Department of Physics, University of Konstanz, D-76457 Konstanz, Germany. e-mail: Guido.Burkard@uni-konstanz.de

\section{References}

1. Loss, D. \& DiVincenzo, D. P. Phys. Rev. A 57, 120-126 (1998).

2. Hanson, R., Kouwenhoven, L. P., Petta, J. R., Tarucha, S. \& Vandersypen, L. M. K. Rev. Mod. Phys. 79, 1217-1265 (2007).

. DiVincenzo, D. P. Fortschritte der Physik 48, 771-783 (2000).

4. Pei, F., Laird, E. A., Steele, G. A. \& Kouwenhoven, L. P. Nature Nanotech. 7, 630-634 (2012).

5. Pályi, A. \& Burkard, G. Phys. Rev. B 82, 155424 (2010).

6. Churchill, H. O. H. et al. Nature Phys. 5, 321-326 (2009).

7. Churchill, H. O. H. et al. Phys. Rev. Lett. 102, 166802 (2009).

8. Kuemmeth, F., Ilani, S., Ralph, D. C. \& McEuen, P. L. Nature 452, 448-452 (2008)

9. Flensberg, K. \& Marcus, C. M. Phys. Rev. B 81, 195418 (2010) 10. Pályi, A. \& Burkard, G. Phys. Rev. Lett. 106, 086801 (2011).

\title{
A discerning look at the bonds in a molecule
}

The resolution of a scanning probe microscope can be improved by attaching a small molecule to the tip of a probe and this has previously allowed individual atoms and bonds within adsorbed organic molecules to be resolved. Researchers at IBM's Zurich Research Laboratory, Universidade de Santiago de Compostela and CEMES-CNRS in Toulouse have now shown that an atomic force microscope (AFM) with a carbon monoxide molecule on its tip can also be used to discriminate between different types of chemical bonds (Science 337, 1326-1329; 2012)

Leo Gross and colleagues imaged $\mathrm{C}_{60}$ molecules and polycyclic aromatic hydrocarbons adsorbed on a copper surface and found that carbon-carbon bonds within the molecules had different contrasts and apparent lengths, features that could be correlated with their bond orders. The images are formed by measuring the change in resonant

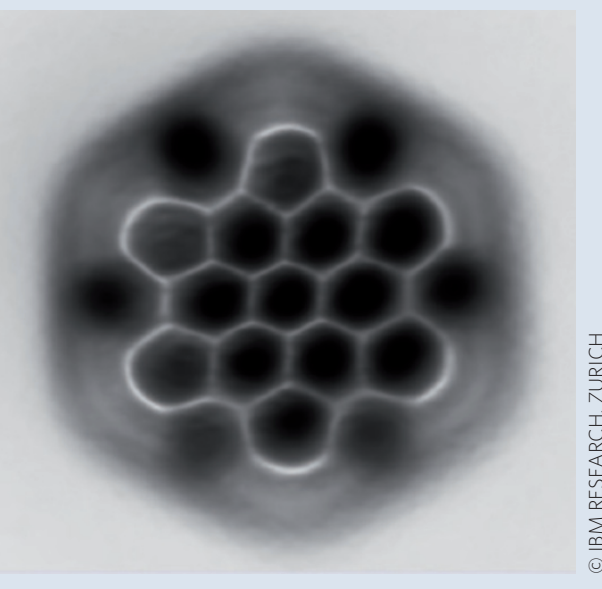

brighter due to a stronger Pauli repulsion between the tip and the sample. The shorter length of such bonds could also be distinguished because the $\mathrm{CO}$ molecules at the apex of the tip tilt during imaging, which amplifies the apparent differences in length.

The AFM image (pictured), which measures approximately $20 \AA$ across, shows a polycyclic aromatic hydrocarbon composed of 13 fused benzene rings. The bonds of the ring at the centre of the planar molecule are of greater bond order than the bonds connecting the central ring to the outer rings, and accordingly appear brighter and have a shorter apparent length. The bonds can be distinguished despite the fact that they differ in length by only $0.03 \AA$.

frequency, or frequency shift, of the oscillating cantilever on which the tip is mounted: repulsive forces create a positive frequency shift, attractive forces a negative shift. Bonds with a higher bond order, which have a greater electron density, appeared
(The bonds at the periphery of the molecule are subject to a variety of effects, which obscure the bond-order differences.)

OWAIN VAUGHAN 